\title{
Extended endoscopic endonasal transclival clipping of posterior circulation aneurysms - an alternative to the transcranial approach
}

\author{
Mohamed Labib ${ }^{1,2} \cdot$ Amir R. Dehdashti ${ }^{1,2}$
}

Received: 4 October 2015 / Accepted: 7 October 2015 /Published online: 21 October 2015

(C) Springer-Verlag Wien 2015

Somanna et al. present a small series of four patients with basilar (BA) aneurysms who were treated partially or entirely using a purely endoscopic endonasal approach (EEA). The authors are to be commended for their brave attempt at clipping these rather complex aneurysms through the transsphenoidal transclival exposure. They should also be praised for the honest reporting of their results. Let's start by emphasizing that the authors of this editorial are strong advocates of endoscopic endonasal skull base surgery and the lead author (AD) has significant surgical experience with complex skull base pathologies treated via an EEA. However, we have also published on the limitations of endoscopic endonasal surgery $[1,2]$ and believe that the enthusiasm for EEA in cerebrovascular surgery should be evaluated very cautiously so as not to compromise patient outcomes. Furthermore, application of EEA in cerebrovascular surgery, specifically in the current endovascular era, could potentially be detrimental to both expanded endocsopic endonasal surgery and open cerebrovascular surgery fields if similar outcomes are not achieved.

Acknowledging the lack of access to endovascular treatment in some countries, we will hereby outline our criticism.

First of all, the case selection process was suboptimal. The authors, rightfully, emphasized the importance of the "midline" positioned basilar artery (BA) but overlooked the significance of the level of the BA bifurcation. Three of the patients presented had "high" bifurcations with the neck above the dorsum sella and the dome certainly well above that. This

Amir R. Dehdashti

Adehdashti@NSHS.edu

1 Hofstra Northshore LIJ School of Medicine, Department of Neurosurgery, Northshore University Hospital, Manhasset, NY, USA

2 Lenox Hill Hospital, New York, NY, USA necessitated drilling of the dorsum sella and posterior clinoid as well as a "pituitary transposition" to reach out behind the dorsum. Even after achieving the after-mentioned exposure and with the aid of the angled $30^{\circ}$ scope, they were not able to properly visualize the perforator-rich area at the posterior aspect of the BA bifurcation. In fact, even if the perforators were visualized, dissecting them properly off the neck/dome of the aneurysm would have been difficult given the present instrumentation. This constellation of limitations in access, optics, and instrumentation resulted in perforator injuries in three of the four patients. These challenges are not surprising given that some of them have already been demonstrated $[3,4]$. A cadaveric study has shown that the endoscopic endonasal exposure of the BA bifurcation was not possible in $36 \%$ of the specimens (and we certainly realize that in that study pituitary transposition was not performed) [3]. But even when the transposition is performed and the basilar apex is at or below the level of the dorsum sella, perforator injuries using this approach remain significant [4]. The fourth patient however had a basilar trunk aneurysm where a traditional craniotomy approach would have been more challenging, and we believe the endoscopic endonasal approach was effective in this particular case and had a good outcome.

Second, the authors performed three pituitary transpositions in order to access the retrosellar space but failed to comment on the technique of transposition (intradural vs. extradural vs. interdural). More significantly, there was no mention of the postoperative endocrinological status of their patients given the wellrecognized transient and/or most likely permanent pituitary dysfunction associated with transposing the pituitary gland. We believe that this is a major limitation as it may convey to novices an unrealistically low risk profile for this rather complex maneuver.

Third, lateral and anterolateral open transcranial approaches would have been the appropriate option for the first three cases in the absence of the least invasive endovascular 
option. Pterional or orbitozygomatic approaches with a variety of intradural exposures (transsylvian, half and half, and so on) for the treatment of these complex lesions are viable surgical alternatives that have stood the test of time and the scrutiny of the neurosurgical community for the past 40 years. With adequate CSF release, an orbitozygomatic approach could also be performed with the addition of posterior clinoidectomy if needed, exposing the lateral wall of the cavernous sinus, mobilizing the third nerve, and allowing an appropriate proximal and distal control of the BA complex and all perforators. Modern microneurosurgery does not need a large craniotomy or significant brain retraction. Nevertheless, microsurgical treatment of basilar tip region aneurysms remains very challenging.

Last but not least, the overall clinical outcomes in this small series were poor. While there were no mortalities, $75 \%$ of the patients developed new neurological deficits, including $50 \%$ permanent injuries. This is devastating to patients who presented with low-grade SAH (as evidenced by their ability to discuss treatment options preoperatively and consent for the interventions). Furthermore, the CSF leak/meningitis and retreatment rates were $25 \%$ each. These results are certainly inferior to those of recent surgical series. For example, the series reported by Krisht et al. [5] on 50 complex BA aneurysms had no mortalities and a $6 \%$ rate of new permanent neurological deficits. Similarly, Sanai et al. [6] reported on 228 aneurysms of the posterior circulation showing transient and permanent neurological deficits in $11.5 \%$ and $7.8 \%$, respectively. The mortality rate in that series was $7.4 \%$. Our personal unpublished data on surgery for posterior circulation aneurysms in a series of 35 aneurysms (ruptured and unruptured, a mixed population with different initial presentation and grades) reveals an $82 \%$ good outcome (mRS 0-2) and $6 \%$ mortality rate. It does not seem, based on this small series of four patients and the current status of endoscopic endonasal surgery, that this technique is ready for prime time, and it is nowhere close to being compared equally in terms of its efficacy to modern microneurosurgery for intracranial aneurysms even in the posterior circulation. At this point, we tend to believe that a mid-basilar trunk aneurysm is potentially the only reasonable indication for this technique. The EEA in experienced hands is probably an equal choice to craniotomy (if not better) for this particular indication, only in the absence of an endovascular access or treatment.
Minimally invasive neurosurgery does not necessarily mean using an endoscopic endonasal versus a craniotomy approach or using an endoscope versus a microscope; instead, it preaches the execution of each approach in a fashion that maximizes efficiency and minimizes postoperative stroke and other complications. To advocate for no "incision" and no "retraction" at the expense of not visualizing appropriately, manipulating, and protecting important BA perforators sharply contrasts the notion of minimally invasive neurosurgery.

Finally, we would like to point out that the neurosurgical dogma should be carefully scrutinized. Operative neurosurgery is a dynamic and progressive science, and the search for alternative operative routes to minimize brain damage should be recognized and advocated for. The expertise in endoscopic endonasal surgery can only be improved by pushing the envelope and honestly reporting the limitations and complications of this approach. Innovation and creativity are the sine qua non of our philosophy to advance the field of neurosurgery, specifically endoscopic endonasal skull base surgery. We are therefore thankful to Somanna and colleagues for their contribution.

\section{References}

1. Dehdashti AR, Ganna A, Witterick I, Gentili F (2009) Expanded endoscopic endonasal approach for anterior cranial base and suprasellar lesions: indications and limitations. Neurosurgery 64(4): 677-687, discussion 687-9

2. Dehdashti AR, Karabatsou K, Ganna A, Witterick I, Gentili F (2008) Expanded endoscopic endonasal approach for treatment of clival chordomas: early results in 12 patients. Neurosurgery 63(2):299307, discussion 307-9

3. Lai LT, Morgan MK, Chin DC, Snidvongs K, Huang JX, Malek J, Lam M, McLachlan R, Harvey RJ (2013) A cadaveric study of the endoscopic endonasal transclival approach to the basilar artery. J Clin Neurosci 20(4):587-592

4. Gardner PA, Vaz-Guimaraes F, Jankowitz B, Koutourousiou M, Fernandez-Miranda JC, Wang EW, Snyderman CH (2015) Endoscopic endonasal clipping of intracranial aneurysms: surgical technique and results. World Neurosurg 25. doi:10.1016/j.wneu. 2015.06.032

5. Krisht AF (2005) Transcavernous approach to diseases of the anterior upper third of the posterior fossa. Neurosurg Focus 19(2), E2

6. Sanai N, Tarapore P, Lee AC, Lawton MT (2008) The current role of microsurgery for posterior circulation aneurysms: a selective approach in the endovascular era. Neurosurgery 62(6):1236-1249, discussion 1249-53 